EPJ Web of Conferences 59, 07005 (2013)

DOI: $10.1051 /$ epjconf/20135907005

(C) Owned by the authors, published by EDP Sciences, 2013

\title{
Route to 100 TW Ti: Sapphire laser at repetitive mode
}

\author{
Hao Teng ${ }^{1}$, Jinglong Ma ${ }^{1}$, Zhaohua Wang ${ }^{1}$, Yi Zheng ${ }^{1}$, Xulei Ge ${ }^{1}$, Zhiyi Wei ${ }^{1 \text {,a }}$ \\ Yutong $\mathrm{Li}^{1}$ and Jie Zhang ${ }^{1,2}$ \\ ${ }^{1}$ Institute of Physics, Chinese Academy of Sciences, Beijing 100190, China \\ ${ }^{2}$ Department of Physics, Shanghai Jiaotong University, Shanghai 200240, China
}

\begin{abstract}
We demonstrated a $100 \mathrm{TW}$-class femtosecond Ti: sapphire laser running at repetition rate of $0.1 \mathrm{~Hz}$ by adding a stage amplifier in the $20 \mathrm{TW} / 10 \mathrm{~Hz}$ laser facility (XL-II). Pumping the new stage amplifier with the $25 \mathrm{~J}$ green Nd:glass laser, we successfully upgraded the laser energy to $3.4 \mathrm{~J}$ with duration of $29 \mathrm{fs}$, corresponding to a peak power of $117 \mathrm{TW}$.
\end{abstract}

\section{INTRODUCTION}

With the advent of chirped-pulse amplification (CPA) technique [1], remarkable progress on the development of high peak power laser system on table-top scale is achieved, peak power up to multiterawatt based on CPA Tisapphire laser has been widely realized in many groups in the world. In particular, petawatt laser systems also were demonstrated in some large laboratories, for example, 1.5 PW in 440 fs hybrid Ti:sapphire - Nd:glass laser system was developed in LLNL [2], 1.1 PW hybrid OPCPA (optical parametrical CPA) Nd:glass laser was developed in Texas [3], and 0.85 PW in 33 fs Ti: sapphire laser system was developed in Japan [4]. However, these petawatt-class laser facilities work at very low repetition rate which is limited of few-shot per hour or single shot. More recently, Jae Hee Sung et al demonstrated a $1 \mathrm{PW}$ Ti: sapphire laser at $0.1 \mathrm{~Hz}$ with multi-stage amplifiers [5], large space and many pump lasers were used to support the laser operation. For a lot of researches on high-intensity laser-matter interaction, it is necessary to accumulate laser shots and improve the signal to noise ratio for experiment [6]. Although we have realized $20 \mathrm{TW}$ laser output at repetition rate of $10 \mathrm{~Hz}$ [7], similar laser facilities were also reported by domestic and international groups [8-11], for many applications such as generation of exceedingly short bursts of energetic radiation and particles [12], peak power of higher than $100 \mathrm{TW}$ running in a repetitive mode is necessary, therefore, it is still a promising work to develop a compact multi-TW femtosecond laser capable of running at significant repetition rate with economical cost.

In this conference, we report a $100 \mathrm{TW}$ Ti:sapphire laser at $0.1 \mathrm{~Hz}$ based on our previous $20 \mathrm{TW}$ femtosecond laser at $10 \mathrm{~Hz}$ (XL-II laser facility) by adding a stage of amplifier before the compressor. Pumping the final amplifier by a $527 \mathrm{~nm} \mathrm{Nd}$ :glass laser at repetition rate of $0.1 \mathrm{~Hz}$ and optimizing the compressed pulse duration, an output laser energy up to $3.4 \mathrm{~J}$ with pulse duration of $29 \mathrm{fs}$ was obtained, which corresponding to a peak of $117 \mathrm{TW}$.

\section{DESIGN OF BOOSTER AMPLIFIER AND EXPERIMENTAL RESULTS}

The "XL-II" laser facility consists of a home-made femtosecond Ti:sapphire laser oscillator, pulse stretcher, a regenerative amplifier, one stage multi-pass amplifier and a vacuum compressor. It was

\footnotetext{
ae-mail: zywei@iphy.ac.cn
}

This is an Open Access article distributed under the terms of the Creative Commons Attribution License 2.0, which permits unrestricted use, distribution, and reproduction in any medium, provided the original work is properly cited. 


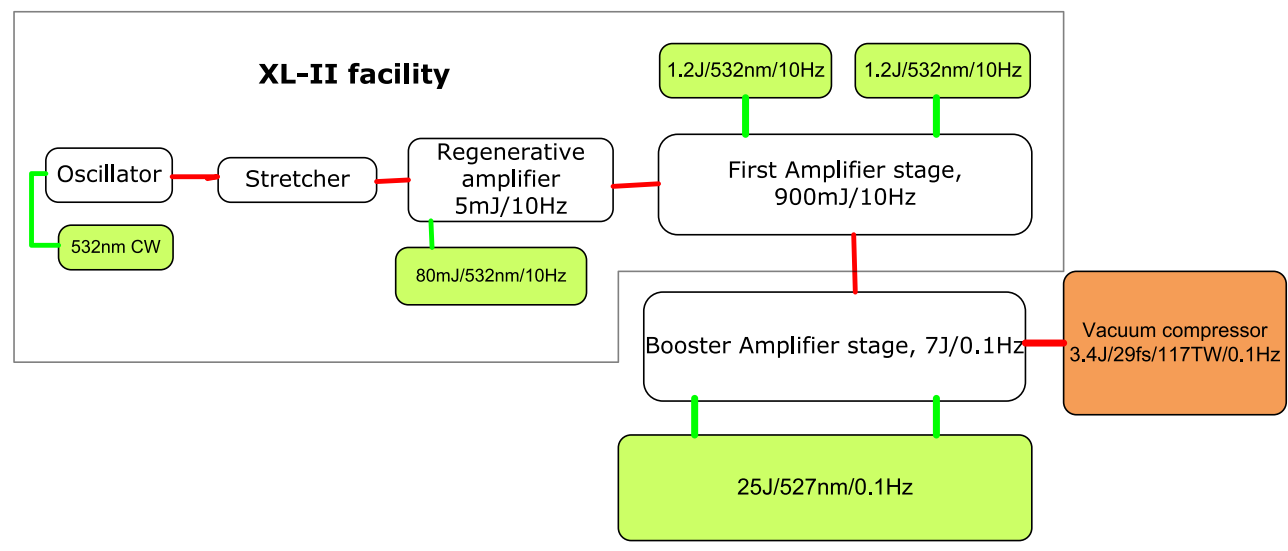

Figure 1. Block diagram of the $117 \mathrm{TW} / 0.1 \mathrm{~Hz}$. laser system.
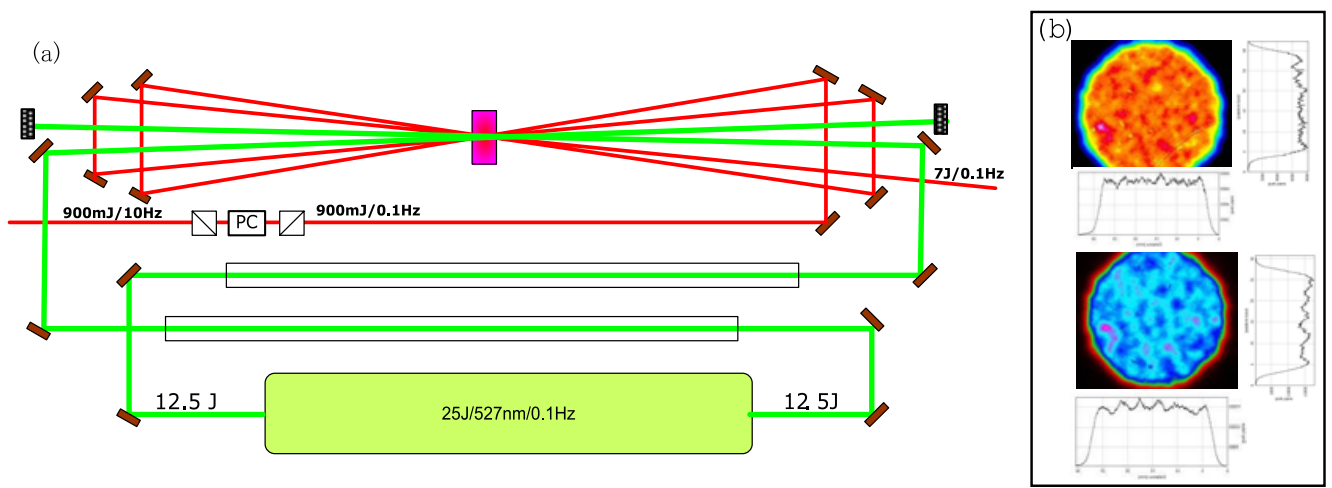

Figure 2. Design of the final booster amplifier (a), spatial profile for the two pump beams (b), which show flat-top spatial distribution.

able to deliver the laser pulses with energy of $600 \mathrm{~mJ}$ in $30 \mathrm{fs}$ at repetition rate of $10 \mathrm{~Hz}$, the detail was described in reference [7]. Based on this 20 TW laser facility, a high energy booster amplifier stage is designed to boost the energy, a commercial $527 \mathrm{~nm} \mathrm{Nd}$ :glass laser with energy of $25 \mathrm{~J}$ at repetition rate of $0.1 \mathrm{~Hz}$ (Thales Inc.) is employed for pumping for last amplifier stage, as shown in figure 1. To realize over $100 \mathrm{TW}$ at high repetition rate, some key techniques must be taken in considerations, for example, homogeneous pump scheme, the thermal effect, the gain narrowing effect and to suppress the parasitic lasing etc.

To achieve good beam quality of compressed beam and improve the extracting efficiency, the homogeneous pump scheme is very important. Two independent oscillators with multimode spatial profile are employed in this pump laser. After combination of these two beams into one beam by thin film polarizer, and injected into two independent amplifiers, by accurately compensating for thermally induced birefringence, spatial modulators in amplifier modules, the near field of beams output from the pump laser are flat-top distribution in spatial domain. By image relay scheme through vacuum tube, this near field beam pattern with flattop distribution is imaged and expanded to $30 \mathrm{~mm}$ in diameter on the Ti: sapphire crystal with diameter of $35 \mathrm{~mm}$ to produce homogeneous pump, which ensures the amplified beam quality and improves the efficiency. The design of booster amplifier is shown in figure 2 .

To suppress the parasitic lasing in larger diameter crystal, a polymer thermoplastic material is cladded around the Ti: sapphire crystal to absorb the reflection at the Ti:sapphhire interface. 
IFSA 2011

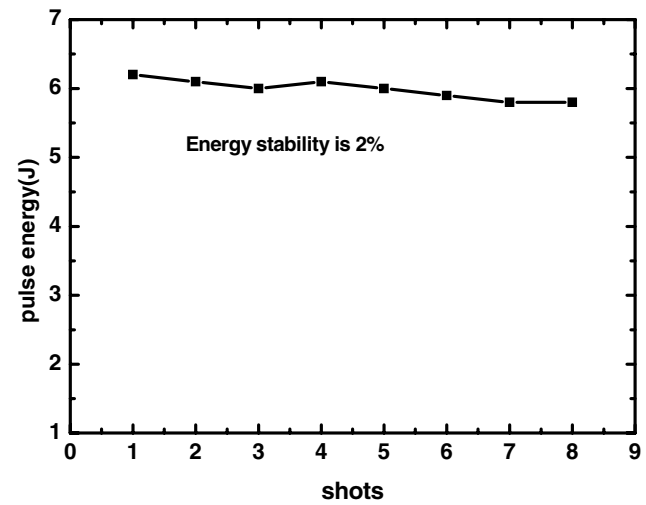

Figure 3. Output energy of booster amplifier pumped with energy of $21 \mathrm{~J}$.

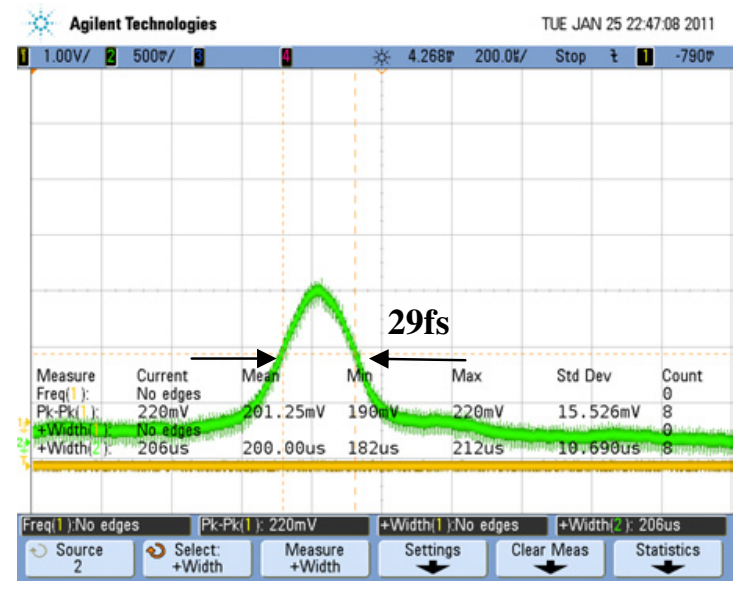

Figure 4. Single shot autocorrelation trace of compressed pulse, which show the pulse duration is $29 \mathrm{fs}$ by calibration.

To compensate the gain narrowing effect and support more bandwidth spectra, a gain notch filter is employed into the regenerative amplifier, with accurately tuning the angle in the beam path of regenerative cavity, the gain narrowing effect will be compensated. The spectrum bandwidth will be enlarged to $32 \mathrm{~nm}$ by inserting gain filter, compared to the $22 \mathrm{~nm}$ without gain filter. It was demonstrated that the gain narrowing effect will be reduced especially in the last booster amplifier stage and support much wider spectral bandwidth.

A Pockels cell with diameter of $30 \mathrm{~mm}$ is located between the first amplifier stage and booster amplifier stage, the repetition rate of amplified pulses can be switched to $0.1 \mathrm{~Hz}$ by this pockels cell and synchronized to the pump laser for the last stage. With fine synchronization and optimized alignment between pump and chirped laser pulse, we obtained amplified pulse with energy of $6 \mathrm{~J}$ under pump energy of $21 \mathrm{~J}$, which corresponds to an extract efficiency of $28 \%$. The shot-to -shot energy stability is about $2 \%$, as shown in figure 3 .

The amplified laser is enlarged to $80 \mathrm{~nm}$ in diameter by a telescope, and injected into the vacuum compressor. After optimized dispersion compensation, we measured the compressed pulse with a commercial single-shot autocorrelator (Coherent Inc.), as shown in figure 4. By calibration and assuming $\mathrm{Sech}^{2}$-shape temporal profile, the calculated pulse duration is $29 \mathrm{fs}$. The energy is about $3.4 \mathrm{~J}$ after the 


\section{EPJ Web of Conferences}

compressor, corresponding to a peak power of $117 \mathrm{TW}$. The contrast ratio is around $10^{6 \sim 7}$ at $1 \mathrm{~ns}$ time scale, which was measured by a photodiode with picosecond resolution. Based on this progress, we plan to replace the front end with a multi-pass amplifier, which has a contrast ratio up to $10^{10}$. Based on the new scheme, we expect the contrast ratio from last stage will be improved to more than $10^{9}$.

\section{SUMMARY}

In summary, based on our previous $20 \mathrm{TW}$ laser (XL-II laser facility), a booster main amplifier was constructed, which is pumped by a green laser with energy of $25 \mathrm{~J}$ at $0.1 \mathrm{~Hz}$. By suppressing the parasitic lasing in Ti: sapphire crystal and employing a homogenous pumping scheme, the amplified energy is boosted to $6 \mathrm{~J}$ before compressor under the pump energy of $21 \mathrm{~J}$, corresponding to an extract efficiency is $28 \%$. By accurately compensating dispersion between the stretcher, amplifier and compressor, and suppressing gain narrowing effect in the regenerative amplifier to got more broader bandwidth, the compressed pulses is as short as $29 \mathrm{fs}$ with energy of $3.4 \mathrm{~J}$, which corresponds to peak power of $117 \mathrm{TW}$. The energy stability is $3 \%$. The laser will pave a way to carry out high field experimental studies at relativistic regime that require repetition rate.

Author thanks for financial support of the National Key Technology R\&D Program of the Ministry of Science and Technology under Grant No. 2012BAC23B03, the National Natural Science Foundation of China under Grant No. 11074298 and No.9112608, the National Basic Research Program of China (973 Program) (No. 2007CB815104), the Instrument Developing Project of the Chinese Academy of Sciences Grant No. 2010004.

\section{References}

[1] D. Strickland and G. Mourou, Opt. Commun. 56, 219 (1985)

[2] M. D. Perry, D. Pennington, B. C. Stuart, G. Tietbohl, J. A. Britten, C. Brown, S. Herman, B. Golick, M. Kartz, J. Miller, H. T. Powell, M. Vergino, and V. Yanovsky, Opt. Lett. 24, 160 (1999)

[3] Erhard Gaul, M. Martinez, J. Blakeney, A. Jochmann, M. Ringuette, D. Hammond, R. Escamilla, W. Henderson, T. Borger, T. Ditmire, International Conference on Ultrahigh Intensity Lasers2008, Oct 27-31, 2008, Tongli, China

[4] M. Aoyama, K. Yamakawa, Y. Akahane, J. Ma, N. Inoue, H. Ueda, and H. Kiriyama, Opt. Lett. 28, 1594 (2003)

[5] Jae Hee Sung, Seong Ku Lee, Tae Jun Yu, Tae Moon Jeong, and Jongmin Lee, Opt. Lett. 35, 3021 (2010)

[6] G. Mourou, T. Tajima, S. V. Bulanov, Rev. Mod. Phys. 78, 309 (2006)

[7] Z.Y. Wei, Z.H. Wang, W.J. Ling, P. Wang, J. Zhang, M. Suzuki, H. Kuroda, X-Ray Lasers 2004, Inst. Phys. Conf.Ser.No. 186, 685 (2004)

[8] H.S. Peng, W.Y. Zhang, Journal of the Korean Physical Society 49, 305 (2006)

[9] XY Liang, YX Leng, LH Lin, HH Lu, WY Wang, YH Jiang, B Shuai, HL Peng, BZ Zhao, C Wang, WQ Zhang, ZQ Zhang, RX Li, ZZ Xu, Opt and Lasers in Engineering 44, 1302006 (2006)

[10] Z.Y.Wei, Z.H.Wang, P.Wang, W.J.Ling, J.F.Zhu, H.N.Han, and J. Zhang, The fifth International Conference on Inertial Fusion Sciences and Applications (IFSA2007), Journal of Physics: Conference Series 112, 032003 (2008)

[11] V.V. Lozhkarev, G. I. Freidman, V. N, Ginzburg, E. V. Katin, E. A. Khazanov, A. V. Kirsanov, G. A. Luchinin, A. N. Mal'shakov, M. A. Martyanov, O. V. Palashov, A.K. Poteomkin, A.M. Sergeev, A.A. Shaykin, I.V. Yakovlev, Laser Phy. Lett. 4, 421 (2007)

[12] G. Mourou, T. Tajima, Science 331, 41 (2011) 